\title{
Quelques propriétés des estimateurs à noyaux gamma pour des échantillons de petites tailles
}

\author{
Mouloud Cherfaoui $^{\dagger, 1}$, Mohamed Boualem ${ }^{\S}$, Djamil Aïssani ${ }^{\ddagger}$ and Smaïl Adjabi ${ }^{\ddagger}$ \\ ${ }^{\dagger}$ Département de Mathématiques, Université de Biskra, 07000 Algérie \\ Unité de Recherche LaMOS (Modélisation et Optimisation des Systèmes), Université de Bejaia, \\ 06000 Bejaia, Algérie \\ ${ }^{\S}$ Unité de Recherche LaMOS (Modélisation et Optimisation des Systèmes), Faculté de Technologie, \\ Université de Bejaia, 06000 Bejaia, Algérie \\ ${ }^{\ddagger}$ Unité de Recherche LaMOS (Modélisation et Optimisation des Systèmes), Faculté des Sciences \\ Exactes, Université de Bejaia, 06000 Bejaia, Algérie
}

Received January 31, 2015; Accepted October 19, 2015

Copyright (C) 2015, Afrika Statistika. All rights reserved

\begin{abstract}
In this paper, we study the nonunit mass of the gamma (gamma and the modified gamma) kernels estimator for the finite samples. For this, we propose to use the normalization approach (Macro-normalization and the Micro-Normalization) to correct the problem of the nonunit mass. In the aim to illustrate the necessity of the normalization approach a detailed simulation study investigates the local and global performances of the considered estimators. The obtained results show the good performances of the normalized gamma kernels estimators.

Résumé. Dans ce papier, nous avons abordé le problème de la non conservation de masse des estimateurs à noyaux gamma (gamma et gamma modifié) pour des échantillons finis. Pour cela, deux approches (Macro-normalisation et Micro-normalisation) ont été proposées pour remédier à ce problème. Afin d'illustrer la nécessité de ces approches, une étude de comparaison par simulation a été réalisée. Par conséquent, les résultats obtenus montrent qu'il est préférable d'utiliser les estimateurs à noyaux gamma normalisés.
\end{abstract}

Key words: Propriété d'unité de masse; Approches de normalisation; Simulation.

AMS 2010 Mathematics Subject Classification : 62G07; 62G20; 62M05.

\footnotetext{
${ }^{1}$ Corresponding author Mouloud Cherfaoui: mouloudcherfaoui2013@gmail.com Mohamed Boualem : mohandboualem@gmail.com

Djamil Aïssani : lamos_bejaia@hotmail.com

Smaïl Adjabi : adjabi@hotmail.com
} 
M. Cherfaoui, M. Boualem, D. Aïssani and S. Adjabi, Afrika Statistika, Vol. 10(1), 2015, pages 763-776. Quelques propriétés des estimateurs à noyaux gamma pour des échantillons de petites tailles.

\section{Introduction}

Soit $X_{1}, X_{2}, \ldots, X_{n}$ un $n$-échantillon issu d'une densité de probabilité inconnue $f$ qui est définie sur un support positif $\left(\left[0, \infty[)\right.\right.$ et deux fois continûment dérivable $\left(f \in \mathcal{C}^{2}([0, \infty[))\right.$. Parmi les estimateurs proposés pour cette famille de distributions, on cite l'estimateur de Chen (2000) qui suggère de remplacer l'estimateur classique de Parzen-Rosenblatt (voir Parzen, 1962 et Rosenblatt, 1956) par:

$$
\hat{f}(x)=\frac{1}{n} \sum_{i=1}^{n} K_{\left(\rho_{h}(x), h\right)}\left(X_{i}\right),
$$

où, $h=h(n)$ représente le paramètre du lissage satisfaisant les conditions $h \rightarrow 0$ et $n h \rightarrow$ $\infty$ lorsque $n \rightarrow \infty$ et $K_{\left(\rho_{h}(x), h\right)}$ est la fonction de densité de la distribution gamma de paramètres $\left(\rho_{h}(x), h\right)$, donnée par la formule suivante:

$$
K_{\left(\rho_{h}(x), h\right)}(t)=\frac{t^{\rho_{h}(x)-1} e^{-t / h}}{h^{\rho_{h}(x)} \Gamma\left(\rho_{h}(x)\right)},
$$

avec

$$
\Gamma(p)=\int_{0}^{\infty} x^{p-1} e^{-x} d x, \quad p>0 .
$$

La première version de l'estimateur à noyau gamma, notée $\hat{f}_{1}(x)$, est obtenue en remplaçant $\rho_{h}(x)$ par $x / h+1$, dans la formule (1) (voir Chen, 2000). Le biais et la variance asymptotiques de $\hat{f}_{1}(x)$ sont donnés respectivement par:

$$
\begin{gathered}
\operatorname{Biais}\left(\hat{f}_{1}(x)\right)=h\left\{f^{\prime}(x)+\frac{1}{2} x f^{\prime \prime}(x)\right\}+o(h), \\
\operatorname{Var}\left\{\hat{f}_{1}(x)\right\}=n^{-1} \frac{h^{-1} \Gamma(2 x / h+1)}{2^{2 x / h+1} \Gamma^{2}(x / h+1)} f(x)+o\left(n^{-1}\right) .
\end{gathered}
$$

En raison de la contribution indésirable de $f^{\prime}$ dans le biais de l'estimateur $\hat{f}_{1}(x)$ (voir l'expression du biais donnée par la formule (2)). Une autre version de $\hat{f}_{1}(x)$ appelée estimateur à noyau gamma modifié, notée $\hat{f}_{2}(x)$, est obtenue en remplaçant $\rho_{h}(x)$, dans la formule (1), par la nouvelle quantité suivante:

$$
\begin{aligned}
\rho_{h}(x) & =(x / h) \mathbb{I}_{\{x \geq 2 h\}}+\left(\frac{1}{4}(x / h)^{2}+1\right) \mathbb{I}_{\{x \in[0,2 h[\}} \\
& = \begin{cases}x / h, & \text { si } x \geq 2 h, \\
\frac{1}{4}(x / h)^{2}+1, & \text { si } x \in[0,2 h[.\end{cases}
\end{aligned}
$$

Le biais asymptotique de $\hat{f}_{2}(x)$ est exprimé par la formule suivante:

$$
\text { Biais }\left\{\hat{f}_{2}(x)\right\}= \begin{cases}\frac{h}{2} x f^{\prime \prime}(x), & \text { si } x \geq 2 h, \\ h \xi_{h}(x) f^{\prime}(x), & \text { si } x \in[0,2 h[,\end{cases}
$$

où,

$$
\xi_{h}(x)=(1-x)\left\{\rho_{h}(x)-x / h\right\} /\left\{1+h \rho_{h}(x)-x\right\} .
$$


M. Cherfaoui, M. Boualem, D. Aïssani and S. Adjabi, Afrika Statistika, Vol. 10(1), 2015, pages 763-776. Quelques propriétés des estimateurs à noyaux gamma pour des échantillons de petites tailles.

Notons que la variance de $\hat{f}_{2}(x)$ est similaire à celle de $\hat{f}_{1}(x)$ (voir Chen, 2000).

La forme et la qualité du lissage des estimateurs à noyaux gamma changent selon la position où la densité est estimée, ce qui fait la différence avec les autres noyaux (noyaux symétriques). Ils sont exempts de problème du biais aux bornes, non négatif et réalisent un taux de convergence optimal pour l'erreur carrée intégrée moyenne (MISE). Également, ils atteignent le taux de convergence optimal pour les variables i.i.d au sens de MISE dans la classe des estimateurs à noyaux non négatifs. De plus, ils permettent une réduction de la variance lors du lissage en s'éloignant de la borne.

D'autres propriétés des estimateurs à noyaux gamma sont bien documentées dans la littérature. Bouezmarni and Scaillet (2003) ont établi les conditions de convergence faible, de ce dernier, sur un compact $[0,+\infty[$, lorsque $f$ est continue sur ce support ainsi que la convergence faible au sens MIAE (Mean Integer Absolute Error). Pour les densités non bornées à l'origine (au voisinage de zéro), les mêmes auteurs ont examiné les performances de cet estimateur par simulation et ont prouvé la convergence en probabilité. Fernandez and Monteiro (2005) ont établi le théorème central limite pour l'estimateur fonctionnel à noyaux gamma.

Dans ce travail, nous nous intéressons aux propriétés des estimateurs à noyaux gamma pour des échantillons finis. En premier lieu, nous avons abordé le problème de la non conservation de masse de ces estimateurs. En effet, après avoir établis certaines propriétés de l'estimateur $\int_{0}^{\infty} \hat{f}_{j}(x) d x, j=1,2$, pour démontrer que les estimateurs à noyaux gamma ne vérifient pas la propriété de l'unité de masse $\left(\int_{0}^{\infty} \hat{f}_{j}(x) d x \neq 1, j=1,2\right)$, nous avons effectué une étude par simulation pour confirmer la non conservation de masse des estimateurs $\hat{f}_{j}(x), j=1,2$. En deuxième lieu, afin de remédier au problème de la non conservation de masse de ces estimateurs, nous avons utilisé la technique de normalisation (Macro-normalisation et Micronormalisation). De plus, nous avons montré par simulation que la normalisation nous fournit des estimateurs plus performants (localement (Biais, Var, MSE) et globalement (ISE moyen)) que l'estimateur $\hat{f}_{j}(x), j=1,2$.

Le reste du document est organisé comme suit: la deuxième section est consacrée essentiellement au problème de la non conservation de masse des noyaux gamma dans le cas des échantillons finis et également on donne certaines propriétés des estimateurs à noyaux gamma et gamma modifié. De plus, un algorithme de simulation est conçu pour illustrer les résultats analytiques obtenus. Dans la troisième section, nous nous intéressons au problème de la correction de la non conservation de masse des estimateurs obtenus par les noyaux gamma. En outre, une étude par simulation Monté Carlo a été réalisée afin de constater l'impact et la nécessité de la normalisation de ces estimateurs. Afin d'illustrer la nécessité de la normalisation, un exemple d'application sur les chânes de Markov est présenté dans la section 4 .

\section{Non conservation de masse des noyaux gamma}

Dans cette section, nous allons discuter le problème de la non conservation de masse des estimateurs à noyaux gamma dans le cas des échantillons de petites tailles.

Il est important de souligner qu'une méthode d'estimation non paramétrique est appropriée si elle satisfait les trois propriétés suivantes: 
M. Cherfaoui, M. Boualem, D. Aïssani and S. Adjabi, Afrika Statistika, Vol. 10(1), 2015, pages 763-776. Quelques propriétés des estimateurs à noyaux gamma pour des échantillons de petites tailles.

1) Fournit un estimateur de la fonction de répartition en échantillon de taille finie (fonction croissante vérifiant la propriété d'unité de masse).

2) Donne un estimateur de la fonction de répartition convergent.

3) Construit un estimateur de la fonction de densité.

Par ailleurs, il est nécessaire de vérifier que les estimateurs fournis par les noyaux gamma, dans le cas des échantillons de petites tailles, vérifient les propriétés précédentes. Particulièrement, on propose d'évaluer les caractéristiques de l'intégrale $I_{j}\left(I_{j}=\int_{0}^{\infty} \hat{f}_{j}(x) d x, j=\right.$ 1,2.) afin de répondre essentiellement à la propriété une.

\subsection{Caractéristiques de l'intégrale $I_{j}$}

Soit $X_{1}, X_{2}, \ldots, X_{n}$ un $n$-échantillon de petite taille $(n<<\infty)$ issu d'une densité de probabilité inconnue $f$ qui est définie sur un support positif et deux fois continûment dérivable $\left(f \in \mathcal{C}^{2}([0, \infty[))\right.$. Par ailleurs, cette densité est estimée par l'un des deux noyaux gamma $\left(\hat{f}_{j}(x), j=1,2\right)$. Dans ce cas, l'intégrale $I_{j}=\int_{0}^{\infty} \hat{f}_{j}(x) d x, j=1,2$, est une variable aléatoire qui dépend de l'échantillon observé, qu'on notera par $I_{j}$. Les résultats suivants donnent certaines propriétés de $I_{j}, j=1,2$, à savoir: son espérance et sa variance.

Proposition 1. L'espérance et la variance de l'intégrale de l'estimateur à noyau gamma $\left(I_{1}\right)$, sont données respectivement par:

$$
\begin{gathered}
\mathbb{E}\left(I_{1}\right)=1+\frac{h}{2}\left[f(x)+x f^{\prime}(x)\right]_{0}^{\infty}+o(h) . \\
\operatorname{Var}\left(I_{1}\right)=\frac{1}{2 \sqrt{\pi}} n^{-1} h^{-1 / 2} \int_{0}^{\infty} x^{-1 / 2} f(x) d x+o\left(n^{-1} h^{-1 / 2}\right) .
\end{gathered}
$$

Preuve. Pour montrer la formule (3), on a

$$
\begin{aligned}
\mathbb{E}\left(I_{1}\right) & =\mathbb{E}\left(\int_{0}^{\infty} \hat{f}_{1}(x) d x\right)=\int_{0}^{\infty} \mathbb{E}\left(\hat{f}_{1}(x)\right) d x \\
& =\int_{0}^{\infty}\left(f(x)+h\left\{f^{\prime}(x)+\frac{1}{2} x f^{\prime \prime}(x)\right\}+o(h)\right) d x \\
& =\int_{0}^{\infty} f(x) d x+h\left\{\int_{0}^{\infty} f^{\prime}(x) d x+h \int_{0}^{\infty} \frac{1}{2} x f^{\prime \prime}(x) d x\right\}+o(h) \\
& =1+h\{\underbrace{\int_{0}^{\infty} f^{\prime}(x) d x}_{Q_{1}}+\frac{1}{2} \underbrace{\int_{0}^{\infty} x f^{\prime \prime}(x) d x}_{Q_{2}}\}+o(h) .
\end{aligned}
$$

D'autre part, on a:

$$
Q_{1}=\int_{0}^{\infty} f^{\prime}(x) d x=[f(x)]_{0}^{\infty} \quad \text { et } \quad Q_{2}=\int_{0}^{\infty} x f^{\prime \prime}(x) d x=\left[x f^{\prime}(x)-f(x)\right]_{0}^{\infty} .
$$

En injectant les quantités de $Q_{1}$ et $Q_{2}$ dans l'équation (5), on obtient la formule (3). 
M. Cherfaoui, M. Boualem, D. Aïssani and S. Adjabi, Afrika Statistika, Vol. 10(1), 2015, pages 763-776. Quelques propriétés des estimateurs à noyaux gamma pour des échantillons de petites tailles.

Pour montrer la formule (4), on a

$$
\begin{aligned}
\operatorname{Var}\left(I_{1}\right) & =\operatorname{Var}\left(\int_{0}^{\infty} \hat{f}_{1}(x) d x\right)=\int_{0}^{\infty} \operatorname{Var}\left(\hat{f}_{1}(x)\right) d x \\
& =\frac{1}{2 \sqrt{\pi}} n^{-1} h^{-1 / 2} \int_{0}^{\infty} x^{-1 / 2} f(x) d x+o\left(n^{-1} h^{-1 / 2}\right) .
\end{aligned}
$$

Proposition 2. L'espérance et la variance de l'intégrale de l'estimateur à noyau gamma modifié $\left(I_{2}\right)$, sont données respectivement par:

$$
\begin{gathered}
\mathbb{E}\left(I_{2}\right)=1+h \int_{0}^{2 h} \xi_{h}(x) f^{\prime}(x) d x+\frac{h}{2}\left[f(x)+x f^{\prime}(x)\right]_{2 h}^{\infty}+o(h) . \\
\operatorname{Var}\left(I_{2}\right)=\frac{1}{2 \sqrt{\pi}} n^{-1} h^{-1 / 2} \int_{0}^{\infty} x^{-1 / 2} f(x) d x+o\left(n^{-1} h^{-1 / 2}\right) .
\end{gathered}
$$

Preuve. Pour montrer la formule (6), on a

$$
\begin{aligned}
\mathbb{E}\left(I_{2}\right) & =\mathbb{E}\left(\int_{0}^{\infty} \hat{f}_{2}(x) d x\right)=\int_{0}^{\infty} \mathbb{E}\left(\hat{f}_{2}(x)\right) d x \\
& =\int_{0}^{\infty}\left(f(x)+\xi_{h}(x) h f^{\prime}(x) \mathbb{I}_{\{x \in[0,2 h[\}}+\frac{1}{2} x f^{\prime \prime}(x) h \mathbb{I}_{\{x \geq 2 h\}}+o(h)\right) d x \\
& =\int_{0}^{\infty} f(x) d x+h \int_{0}^{2 h} \xi_{h}(x) f^{\prime}(x) d x+\frac{h}{2} \int_{2 h}^{\infty} x f^{\prime \prime}(x) d x+o(h) \\
& =1+h\{\int_{0}^{2 h} \xi_{h}(x) f^{\prime}(x) d x+\frac{1}{2} \underbrace{\int_{2 h}^{\infty} x f^{\prime \prime}(x) d x}_{Q}\}+o(h)
\end{aligned}
$$

où,

$$
\xi_{h}(x)=(1-x)\left\{\rho_{h}(x)-x / h\right\} /\left\{1+h \rho_{h}(x)-x\right\} .
$$

D'autre part, on a:

$$
Q=\int_{0}^{\infty} x f^{\prime \prime}(x) d x=\left[x f^{\prime}(x)-f(x)\right]_{2 h}^{\infty} .
$$

En injectant la quantité $Q$ dans l'équation (8), on obtient la formule (6).

La preuve de la formule (7) est similaire à celle de la proposition 1 (voir la formule (4)).

L'exemple suivant donne une illustration de la proposition 1.

Exemple 1. Soit $f$ est une densité de probabilité exponentielle du paramètre $\lambda$. Pour cela, on a:

$$
f(x)=\lambda e^{-\lambda x}, \quad f^{\prime}(x)=-\lambda^{2} e^{-\lambda x} \text { et } f^{\prime \prime}(x)=\lambda^{3} e^{-\lambda x} .
$$

Alors, les expressions de $\mathbb{E}\left(I_{1}\right)$ et $\operatorname{Var}\left(I_{1}\right)$, sont données par:

$$
\begin{gathered}
\mathbb{E}\left(I_{1}\right)=1-\frac{1}{2} h \lambda+o(h) . \\
\operatorname{Var}\left(I_{1}\right)=\frac{1}{2 n} \sqrt{\frac{\lambda}{h}}+o\left(n^{-1} h^{-1 / 2}\right) .
\end{gathered}
$$


M. Cherfaoui, M. Boualem, D. Aïssani and S. Adjabi, Afrika Statistika, Vol. 10(1), 2015, pages 763-776. Quelques propriétés des estimateurs à noyaux gamma pour des échantillons de petites tailles.

Remarque 1. À partir de l'expression (3), on constate que l'estimateur précédent $\hat{f}_{1}$ ne satisfait pas la propriété 1) énoncée dans la Section $2(n<<\infty)$. Ce qui signifie qu'il peut être un estimateur qui ne vérifie pas la propriété d'unité de masse. Plus précisément, on remarque clairement, d'après l'expression (10), que l'estimateur $\hat{f}_{1}$ vérifie la propriété d'unité de masse si et seulement si $h$ tend vers zéro, c'est-à-dire si $n$ tend vers l'infinie.

\subsection{Application numérique et discussion des résultats}

Notre objectif est d'illustrer les résultats analytiques obtenus, dans la Section 2.1, par un exemple numérique appliqué à des échantillons générés à partir d'une distribution de probabilité exponentielle du paramètre $\lambda$. Pour ce faire, nous avons conçu un algorithme de simulation dont les étapes sont:

Étape 1: Générer $X_{i}^{(k)} n$-échantillons issus d'une loi de probabilité cible pour $k=$ $1,2, \ldots, m c$,

Étape 2: Calculer le paramètre du lissage $h_{j}^{*}, j=1,2$, qui minimise le $I S E_{j}$ moyen, $j=$ 1,2 ,

Étape 3: Calculer l'estimateur $\hat{f}_{j}(x), j=1,2$, associé à $h_{j}^{*}, j=1,2$ pour les $m c$ échantillons,

Étape 4: Calculer l'intégrale $I_{j}^{(k)}, j=1,2$, et $k=1,2, \ldots, m c$,

Étape 5: Déterminer le $\min \left\{I_{j}^{(k)}\right\}, \max \left\{I_{j}^{(k)}\right\}, k=1,2, \ldots, m c$ et $\mathbb{E}\left(I_{j}\right)=\frac{1}{m c} \sum_{k=1}^{m c} I_{j}^{(k)}$, $j=1,2$.

L'exécution du programme associé à cet algorithme, implémenté sous environnement Matlab, pour les paramètres d'entrés fixés comme suit: $\lambda=1$, nombre de replications $m c=1000$ et pour différentes tailles $n$, nous a fourni les résultats numériques rangés dans la Tableau 1. Les mêmes résultats sont représentés dans la Figure 1.

\begin{tabular}{ccccccc}
\hline & \multicolumn{3}{c}{$I_{1}^{(k)}$} & \multicolumn{3}{c}{$I_{2}^{(k)}$} \\
\cline { 2 - 7 }$n$ & $\min \left(I_{1}\right)$ & $\operatorname{moy}\left(I_{1}\right)$ & $\max \left(I_{1}\right)$ & $\min \left(I_{2}\right)$ & $\operatorname{moy}\left(I_{2}\right)$ & $\max \left(I_{2}\right)$ \\
\hline 10 & 0.6131 & 0.8180 & 0.9343 & 1.0529 & 1.1135 & 1.1887 \\
50 & 0.8092 & 0.8833 & 0.9257 & 1.0547 & 1.0767 & 1.1008 \\
100 & 0.8720 & 0.9054 & 0.9319 & 1.0429 & 1.0625 & 1.0837 \\
250 & 0.9184 & 0.9371 & 0.9567 & 1.0344 & 1.0433 & 1.0537 \\
500 & 0.9428 & 0.9500 & 0.9578 & 1.0269 & 1.0340 & 1.0400 \\
1000 & 0.9507 & 0.9585 & 0.9640 & 1.0290 & 1.0325 & 1.0367 \\
2500 & 0.9704 & 0.9736 & 0.9754 & 1.0178 & 1.0198 & 1.0227 \\
\hline \hline
\end{tabular}

Tableau 1. Variation des propriétés de $I_{j}$ en fonction de $n, j=1,2$.

À partir de ces résultats (numériques ou graphiques), on constate que: 
M. Cherfaoui, M. Boualem, D. Aïssani and S. Adjabi, Afrika Statistika, Vol. 10(1), 2015, pages 763-776. Quelques propriétés des estimateurs à noyaux gamma pour des échantillons de petites tailles.
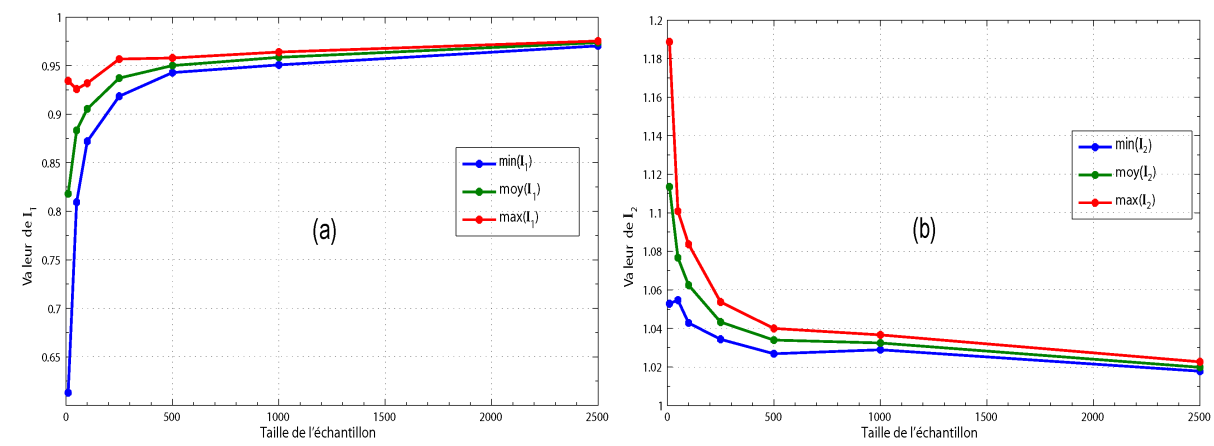

Figure. 1. Courbes de variation des propriétés de $I_{j}$ en fonction de $n, j=1,2$.

- Les estimateurs à noyaux gamma ne vérifient pas la propriété d'unité de masse. En effet, même pour une taille d'échantillon $n$ assez grande:

- La quantité $\int_{0}^{\infty} \hat{f}_{1}(x) d x$ est inférieure à $1\left(\int_{0}^{\infty} \hat{f}_{1}(x) d x<1\right)$ et cela reste vraie même dans le cas idéal (voir la colonne de $\max \left(I_{1}\right)$ ),

- La quantité $\int_{0}^{\infty} \hat{f}_{2}(x) d x$ est supérieure à $1\left(\int_{0}^{\infty} \hat{f}_{2}(x) d x>1\right)$ et cela reste vraie même dans le cas idéal (voir la colonne de $\min \left(I_{2}\right)$ ),

- D'après la formule (10) et comme les paramètres $h$ et $\lambda$ sont positifs, on déduit que $\mathbb{E}\left(I_{1}\right) \leq 1$. Cette dernière inégalité coïncide avec nos résultats de simulation (voir la colonne de $\left.\operatorname{moy}\left(I_{1}\right)\right)$.

Par conséquent, le problème de la non conservation de masse des estimateurs à noyaux gamma peut être corrigé, en utilisant la technique de normalisation qui fera l'objet de la Section suivante.

\section{Normalisation des noyaux gamma}

Dans cette Section, nous nous intéressons au problème de la correction de la non conservation de masse des estimateurs obtenus par les noyaux gamma. Pour ce faire, on utilise la technique de normalisation dont l'idée est de remplacer $\hat{f}_{1}$ (respectivement $\hat{f}_{2}$ ) par l'une des expressions données par les formules (12) et (14) (respectivement, par les formules (13) et (15)).

- Macro-normalisation

$$
\hat{f}_{M 1}(x)=\frac{\hat{f}_{1}(x)}{\int_{0}^{\infty} \hat{f}_{1}(x) d x}
$$

et

$$
\hat{f}_{M 2}(x)=\frac{\hat{f}_{2}(x)}{\int_{0}^{\infty} \hat{f}_{2}(x) d x}
$$


M. Cherfaoui, M. Boualem, D. Aïssani and S. Adjabi, Afrika Statistika, Vol. 10(1), 2015, pages 763-776. Quelques propriétés des estimateurs à noyaux gamma pour des échantillons de petites tailles.

- Micro-normalisation

$$
\hat{f}_{m 1}(x)=\frac{1}{n} \sum_{i=1}^{n} \frac{K_{(x / b+1, b)}\left(X_{i}\right)}{\int_{0}^{\infty} K_{(x / b+1, b)}\left(X_{i}\right) d x}
$$

et

$$
\hat{f}_{m 2}(x)=\frac{1}{n} \sum_{i=1}^{n} \frac{K_{\left(\rho_{h}(x), h\right)}\left(X_{i}\right)}{\int_{0}^{\infty} K_{\left(\rho_{h}(x), h\right)}\left(X_{i}\right) d x} .
$$

On peut par ailleurs montrer que la Macro-normalisation et la Micro-normalisation sont équivalentes en moyenne. Afin de voir l'impact et la nécessité de la normalisation des deux estimateurs $\hat{f}_{1}$ et $\hat{f}_{2}$, nous comparons leurs propriétés avec celles des estimateurs normalisés $\left(\hat{f}_{M 1}, \hat{f}_{m 1}, \hat{f}_{M 2}\right.$ et $\hat{f}_{m 2}$ ), en se basant sur la simulation Monté Carlo. Pour ce faire, on a implémenté un simulateur sous environment Matlab, muni de deux étapes principales suivantes:

- Calcul des propriétés globales ( $h^{*}$, ISE moyen),

- Calcul des propriétés locales (Biais, variance et MSE).

Ces deux étapes ont été appliquées sur 1000 échantillons, de taille $n$, générés à partir d'une distribution exponentielle du paramètre $\lambda=1$. Cependant, après avoir calculer le paramètre du lissage $\left(h^{*}\right)$ qui minimise le $I S E$ moyen de chaque estimateur $\left(h^{*}=\arg \min _{h}\left(\frac{1}{1000} \sum_{i=1}^{1000} I S E^{(i)}(\hat{f}, f)\right)\right.$, où $I S E^{(i)}(\hat{f}, f)$ correspond au $I S E$ associé au $i$ ième échantillon, $i=1,2, \ldots, 1000)$, on s'est intéressé, par la suite, au calcul de biais, de la variance et de $M S E$ des estimateurs considérés. Pour différentes valeurs de $n$, les résultats obtenus, pour les propriétés globales, sont résumés dans les Tableaux 2 et 3. Concernant les propriétés locales, pour $n=100$, les résultats obtenus, sont présentées dans les Figures 2-4.

\begin{tabular}{rcccccccc}
\hline & \multicolumn{2}{c}{$\hat{f}_{1}$} & & \multicolumn{2}{c}{$\hat{f}_{M 1}$} & & \multicolumn{2}{c}{$\hat{f}_{m 1}$} \\
\cline { 2 - 3 } \cline { 8 - 9 }$n$ & $h^{*}$ & ISE & & $h^{*}$ & ISE & & $h^{*}$ & ISE \\
\hline 10 & 0.2938 & 0.0399 & & 0.5369 & 0.0319 & & 0.9636 & 0.0211 \\
50 & 0.1588 & 0.0158 & & 0.2886 & 0.0108 & & 0.7931 & 0.0070 \\
100 & 0.1128 & 0.0119 & & 0.2162 & 0.0088 & & 0.7786 & 0.0056 \\
250 & 0.0812 & 0.0058 & & 0.1419 & 0.0040 & & 0.6880 & 0.0032 \\
500 & 0.0584 & 0.0037 & & 0.1117 & 0.0026 & & 0.6740 & 0.0025 \\
1000 & 0.0475 & 0.0022 & & 0.0883 & 0.0014 & & 0.6915 & 0.0025 \\
2500 & 0.0301 & 0.0012 & & 0.0562 & 0.0008 & & 0.6729 & 0.0024 \\
\hline
\end{tabular}

Tableau 2. Comparaison des $I S E$ moyens des estimateurs à noyaux gamma et gamma normalisés 
M. Cherfaoui, M. Boualem, D. Aïssani and S. Adjabi, Afrika Statistika, Vol. 10(1), 2015, pages 763-776. Quelques propriétés des estimateurs à noyaux gamma pour des échantillons de petites tailles.

\begin{tabular}{rcccccccc}
\hline & \multicolumn{2}{c}{$\hat{f}_{2}$} & & \multicolumn{2}{c}{$\hat{f}_{M 2}$} & & \multicolumn{2}{c}{$\hat{f}_{m 2}$} \\
\cline { 2 - 3 }$n$ & $h^{*}$ & ISE & & $h^{*}$ & ISE & & $h^{*}$ & ISE \\
\hline 10 & 0.4095 & 0.0474 & & 0.3410 & 0.0439 & & 0.3237 & 0.0516 \\
50 & 0.2291 & 0.0168 & & 0.1994 & 0.0160 & & 0.1652 & 0.0223 \\
100 & 0.1739 & 0.0110 & & 0.1528 & 0.0103 & & 0.1020 & 0.0145 \\
250 & 0.1143 & 0.0053 & & 0.1028 & 0.0053 & & 0.0634 & 0.0093 \\
500 & 0.0835 & 0.0033 & & 0.0761 & 0.0033 & & 0.0366 & 0.0063 \\
1000 & 0.0806 & 0.0018 & & 0.0723 & 0.0019 & & 0.0313 & 0.0039 \\
2500 & 0.0462 & 0.0010 & & 0.0425 & 0.0010 & & 0.0144 & 0.0024 \\
\hline
\end{tabular}

Tableau 3. Comparaison des ISE moyens des estimateurs à noyaux gamma: modifiés et modifiés normalisés

\section{Discussion des résultats}

À partir des résultats numériques (voir Tableaux 2 et 3) et graphiques (voir Figures 2-4), on remarque que:

- La normalisation (Macro-normalisation et Micro-normalisation) de l'estimateur $\hat{f}_{1}$ peut améliorer les performances globales de cet estimateur, dans le cas des échantillons finis (voir Tableau 2). Ses bonnes performances peuvent être expliquées par la réduction considérable de la variance de l'estimateur $\hat{f}_{1}$ (voir Figure 3 (a)). Plus précisément, cette normalisation entraîne la réduction du $M S E$ de l'estimateur (voir Figure 4 (a)), au voisinage de zéro, même si cet estimateur subit une légère augmentation du biais.

- La Macro-normalisation améliore les performances globales de l'estimateur $\hat{f}_{2}$, dans le cas des échantillons finis (voir Tableau 3), même s'il est moins performant au voisinage de la borne (voir Figure 4 (b)). Ses bonnes performances peuvent être expliquées par ses bonnes performances locales à l'intérieur du support (en s'éloignant de la borne). En effet, même si cet estimateur est moins performant, au voisinage de la borne au sens du $M S E$ (voir Figure 4 (b)), à cause de l'augmentation du biais (voir Figure 2 (b)), cette perte reste négligeable, par rapport à l'amélioration de sa qualité, au-delà de voisinage de la borne.

- L'augmentation considérable du biais et de la variance, au voisinage de la borne, causée par la Micro-normalisation de l'estimateur $\hat{f}_{2}$, entraîne la construction d'un nouveau estimateur moins performant globalement.

En guise de conclusion, ces constatations nous permettent de dire que la Macro-normalisation de l'estimateur à noyau gamma nous fournit, en générale, un nouveau estimateur plus performant localement et globalement. Cependant, dans le pire des cas ses performances sont équivalentes à celles de l'estimateur classique à noyau gamma. Tandis que, pour l'estimateur à noyau gamma modifié, il est préférable d'éviter la normalisation le fait que c'est l'estimateur classique qui est plus performant. 
M. Cherfaoui, M. Boualem, D. Aïssani and S. Adjabi, Afrika Statistika, Vol. 10(1), 2015, pages 763-776. Quelques propriétés des estimateurs à noyaux gamma pour des échantillons de petites tailles.
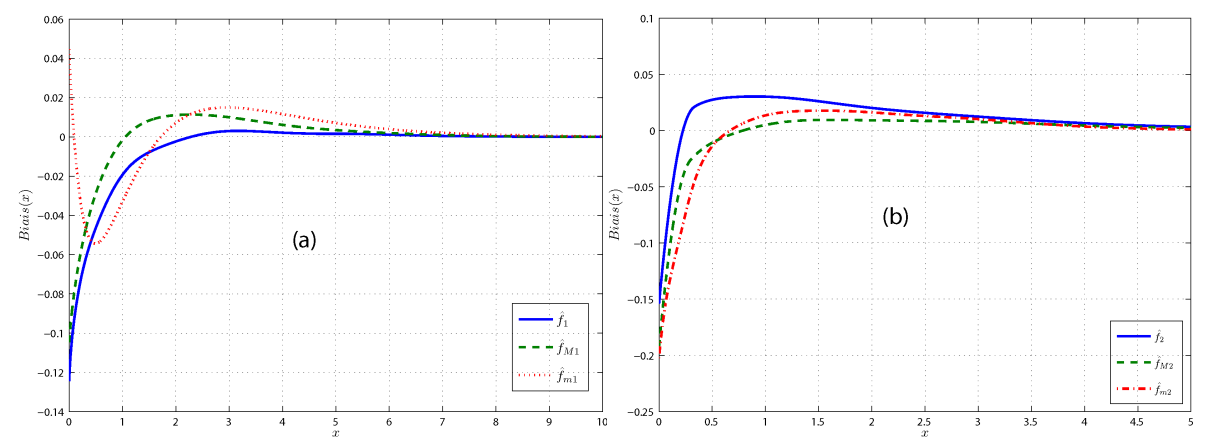

Figure. 2. Variation du biais des estimateurs à noyaux gamma
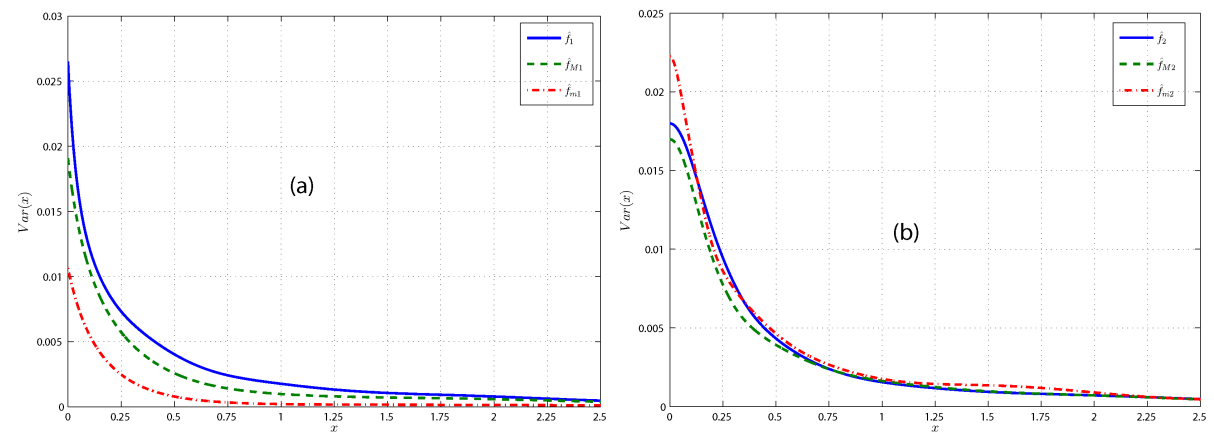

Figure. 3. Variation de la variance des estimateurs à noyaux gamma
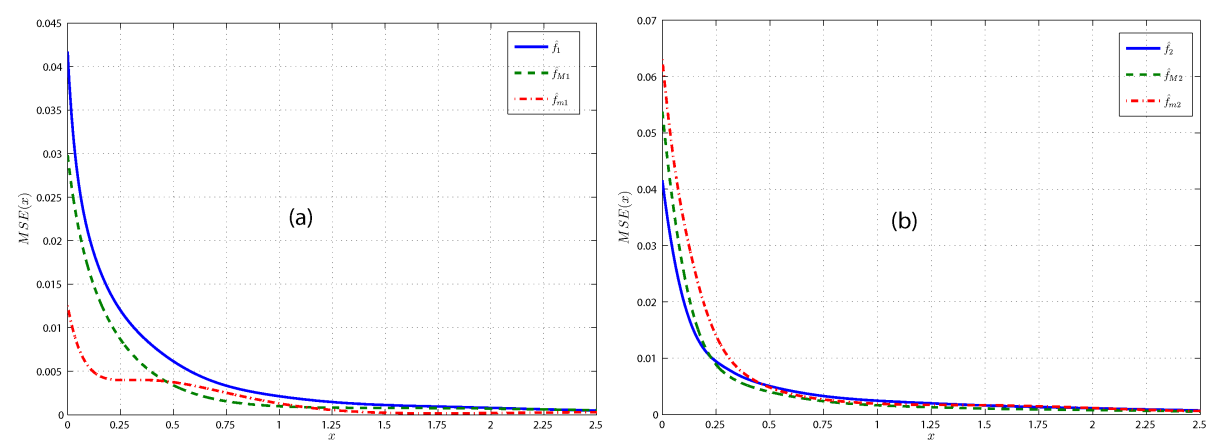

Figure. 4. Variation du MSE des estimateurs à noyaux gamma

\section{Exemple d'application: Estimation d'une matrice de transition}

Maintenant, nous allons illustrer par un exemple d'application les différents estimateurs cités auparavant, afin de confirmer la nécessité de la normalisation des estimateurs à noy- 
M. Cherfaoui, M. Boualem, D. Aïssani and S. Adjabi, Afrika Statistika, Vol. 10(1), 2015, pages 763-776. Quelques propriétés des estimateurs à noyaux gamma pour des échantillons de petites tailles.

aux gamma. En effet, supposons que nous disposons d'un $n$-échantillon $T_{1}, T_{2}, \ldots, T_{n}$ qui représente les durées des inter-arrivées, dans un système d'attente $G I / M / 1 / N$, ayant comme densité de probabilité inconnue $f$ d'une fonction de répartition $F$. L'estimation de la matrice de transition, $\mathbb{P}$, de la chaîne de Markov induite associée au système d'attente $G I / M / 1 / N$ (les mesures de performance explicites de ce système d'attente sont disponibles dans Kleinrock, 1975), donnée par:

$$
P_{i j}= \begin{cases}\int_{0}^{\infty} \frac{e^{-\mu t}(\mu t)^{i-j+1}}{(i-j+1) !} f(t) d t, & \text { si } 1 \leq j \leq i+1 \leq N \\ \int_{0}^{\infty} \frac{e^{-\mu t}(\mu t)^{N-j}}{(N-j) !} f(t) d t, & \text { si } 1 \leq j \leq N \text { et } i=N \\ 1-\sum_{k=1}^{N} P_{i k}, & \text { si } j=0 \\ 0, & \text { sinon, }\end{cases}
$$

consiste à évaluer la densité inconnue $f$ et de substituer son estimateur, dans les $P_{i j}$ (voir Cherfaoui et al., 2015).

Pour répondre à notre objectif, nous avons conçu un algorithme dont les étapes principales sont:

Étape 1: Générer un échantillon de taille $n$ de la loi des inter-arrivées,

Étape 2: Calculer le paramètre du lissage $h^{*}$ qui minimise le $I S E$ moyen, pour chaque estimateurs,

Étape 3: Calculer $\hat{\mathbb{P}}$, en utilisant les différents estimateurs.

Pour l'application numériques nous considérons la situation suivante: $F$ est une loi exponentielle du paramètre $\lambda=1$ (taux des arrivées), $N=5$ (capacité du système), $\mu=1.11$ (taux de service), $m c=100$ (nombre de replications) et $n=20$.

L'exécution de cet algorithme donne les résultats suivants:

- La matrice de transition $\mathbb{P}$ (exacte) de la chaîne de Markov induite:

$$
\mathbb{P}_{\text {Exacte }}=\left(\begin{array}{cccccc}
0.526 & 0.474 & 0 & 0 & 0 & 0 \\
0.277 & 0.249 & 0.474 & 0 & 0 & 0 \\
0.146 & 0.131 & 0.249 & 0.474 & 0 & 0 \\
0.077 & 0.069 & 0.131 & 0.249 & 0.474 & 0 \\
0.040 & 0.036 & 0.069 & 0.131 & 0.249 & 0.474 \\
0.040 & 0.036 & 0.069 & 0.131 & 0.249 & 0.474
\end{array}\right) .
$$

- Noyaux gamma originaux:

$$
\hat{\mathbb{P}}_{\hat{f}_{1}}=\left(\begin{array}{cccccc}
0.595 & 0.406 & 0 & 0 & 0 & 0 \\
0.368 & 0.227 & 0.406 & 0 & 0 & 0 \\
0.242 & 0.126 & 0.227 & 0.406 & 0 & 0 \\
0.172 & 0.070 & 0.126 & 0.227 & 0.406 & 0 \\
0.133 & 0.038 & 0.070 & 0.126 & 0.227 & 0.406 \\
0.133 & 0.038 & 0.070 & 0.126 & 0.227 & 0.406
\end{array}\right)
$$


M. Cherfaoui, M. Boualem, D. Aïssani and S. Adjabi, Afrika Statistika, Vol. 10(1), 2015, pages 763-776. Quelques propriétés des estimateurs à noyaux gamma pour des échantillons de petites tailles.

$$
\hat{\mathbb{P}}_{\hat{f}_{2}}=\left(\begin{array}{cccccc}
0.538 & 0.461 & 0 & 0 & 0 & 0 \\
0.261 & 0.278 & 0.461 & 0 & 0 & 0 \\
0.102 & 0.159 & 0.278 & 0.461 & 0 & 0 \\
0.012 & 0.090 & 0.159 & 0.278 & 0.461 & 0 \\
-0.038 & 0.050 & 0.090 & 0.159 & 0.278 & 0.461 \\
-0.038 & 0.050 & 0.090 & 0.159 & 0.278 & 0.461
\end{array}\right)
$$

- Macro-Normalisation:

$$
\begin{aligned}
& \hat{\mathbb{P}}_{\hat{f}_{M 1}}=\left(\begin{array}{cccccc}
0.571 & 0.429 & 0 & 0 & 0 & 0 \\
0.324 & 0.247 & 0.429 & 0 & 0 & 0 \\
0.183 & 0.141 & 0.247 & 0.429 & 0 & 0 \\
0.102 & 0.081 & 0.141 & 0.247 & 0.429 & 0 \\
0.057 & 0.046 & 0.081 & 0.141 & 0.247 & 0.429 \\
0.057 & 0.046 & 0.081 & 0.141 & 0.247 & 0.429
\end{array}\right), \\
& \hat{\mathbb{P}}_{\hat{f}_{M 2}}=\left(\begin{array}{cccccc}
0.575 & 0.425 & 0 & 0 & 0 & 0 \\
0.322 & 0.253 & 0.425 & 0 & 0 & 0 \\
0.178 & 0.144 & 0.253 & 0.425 & 0 & 0 \\
0.097 & 0.081 & 0.144 & 0.253 & 0.425 & 0 \\
0.053 & 0.045 & 0.081 & 0.144 & 0.253 & 0.425 \\
0.053 & 0.045 & 0.081 & 0.144 & 0.253 & 0.425
\end{array}\right) .
\end{aligned}
$$

- Micro-Normalisation:

$$
\begin{aligned}
\hat{\mathbb{P}}_{\hat{f}_{m 1}}= & \left(\begin{array}{cccccc}
0.557 & 0.443 & 0 & 0 & 0 & 0 \\
0.318 & 0.239 & 0.443 & 0 & 0 & 0 \\
0.184 & 0.134 & 0.239 & 0.443 & 0 & 0 \\
0.107 & 0.077 & 0.134 & 0.239 & 0.443 & 0 \\
0.062 & 0.045 & 0.077 & 0.134 & 0.239 & 0.443 \\
0.062 & 0.045 & 0.077 & 0.134 & 0.239 & 0.443
\end{array}\right), \\
\hat{\mathbb{P}}_{\hat{f}_{m 2}}= & \left(\begin{array}{cccccc}
0.523 & 0.477 & 0 & 0 & 0 & 0 \\
0.280 & 0.243 & 0.477 & 0 & 0 & 0 \\
0.150 & 0.130 & 0.243 & 0.477 & 0 & 0 \\
0.079 & 0.071 & 0.130 & 0.243 & 0.477 & 0 \\
0.041 & 0.038 & 0.071 & 0.130 & 0.243 & 0.477 \\
0.041 & 0.038 & 0.071 & 0.130 & 0.243 & 0.477
\end{array}\right) .
\end{aligned}
$$

À partir des résultats obtenus à l'aide de cet algorithme, on constate que:

L'utilisation de l'estimateur à noyau gamma nous fournis un estimateur, $\hat{\mathbb{P}}_{\hat{f}_{1}}$, non satisfaisant le fait que l'écart entre les valeurs de la matrice de cet estimateur et celles de la matrice exacte est considérable. En effet, cet écart se voit clairement sur les valeurs de la première colonne des deux matrices $\hat{\mathbb{P}}_{\hat{f}_{1}}$ et $\hat{\mathbb{P}}_{\text {Exacte }}$.

On ne peut rien conclure sur la qualité de l'estimateur $\hat{\mathbb{P}}_{\hat{f}_{2}}$, car sa matrice contient des valeurs négatives or les éléments d'une matrice de transition sont tous positifs. 
M. Cherfaoui, M. Boualem, D. Aïssani and S. Adjabi, Afrika Statistika, Vol. 10(1), 2015, pages 763-776. Quelques propriétés des estimateurs à noyaux gamma pour des échantillons de petites tailles.

L'utilisation des estimateurs normalisés nous fournis des estimateurs acceptables et plus performants. Le meilleur estimateur de cette matrice de transition est obtenu par l'utilisation de $\hat{f}_{m 2}$.

Les résultats obtenus dans la Section 3, montrent qu'il est préférable d'éviter la normalisation de l'estimateur à noyau gamma modifié. Quoique cet exemple d'application illustre qu'il est nécessaire, dans certaines situations, de faire recours à cette technique (de normalisation) afin d'éviter des résultats contradictoires.

\section{Conclusion}

Dans cet article, nous avons abordé le problème de la non conservation de masse des estimateurs à noyaux gamma, dans le cas des échantillons finis. Pour ce faire, nous avons élaboré certaines propriétés de ces estimateurs. En outre, nous avons effectué une étude par simulation en vue de valider ces propriétés. Pour remédier au problème de la non conservation de masse des estimateurs à noyaux gamma, deux techniques de normalisation ont été proposées, à savoir la Macro-normalisation et la Micro-normalisation. De plus et afin de justifier l'utilité et la nécessité de ces deux techniques, une comparaison par simulation des performances (locales (Biais, Variance et $M S E$ ) et globales (ISE moyen)) des estimateurs à noyaux gamma et ceux de leurs estimateurs normalisés a été effectuée. Les résultats de comparaison obtenus nous permettent de conclure que la normalisation, des estimateurs à noyaux gamma, est nécessaire. Ce dernier résultat est confirmé par un exemple d'application sur les chaînes de Markov.

La généralisation de cette technique aux estimateurs d'une densité définie sur un support borné, en utilisant d'autres noyaux asymétriques est par conséquent envisageable. De plus, il serait utile de généraliser cette étude sur d'autres densités, avec ou sans pôle. Afin de vérifier l'impact et les performances de la technique de normalisation sur la qualité des estimateurs à la borne, une étude comparative similaire à celle abordée par Zhang (2010), par rapport à d'autres techniques de correction du biais aux bornes, est souhaitable.

\section{Références}

Bouezmarni, T. and Scaillet, O., 2003. Consistency of asymmetric kernel density estimators and smoothed histograms with application to income data. Econometric Theory. 21, 390412 .

Chen, S.X., 2000. Probability density functions estimation using gamma kernels. Annals of the Institute of Statistical Mathematics. 52, 471-480.

Cherfaoui, M., Boualem, M., Aïssani, D. and Adjabi, S., 2015. Choix du paramètre de lissage dans l'estimation à noyau d'une matrice de transition d'un processus semi-markovien. Comptes Rendus Mathématique. 353(3), 273-277.

Fernandez, M. and Monteiro, P., 2005. Central limit theorem for asymmetric kernel functionals. Annals of the Institute of Statistical Mathematics. 57, 425-442.

Kleinrock, L., 1975. Queueing Systems: Theory. Vol. I. John Wiley and Sons (Ed), New York.

Parzen, E., 1962. On estimation of a probability density function and mode. Annals of Mathematical Statistics. 33(3), 1065-1076. 
M. Cherfaoui, M. Boualem, D. Aïssani and S. Adjabi, Afrika Statistika, Vol. 10(1), 2015, pages 763-776. Quelques propriétés des estimateurs à noyaux gamma pour des échantillons de petites tailles.

Rosenblatt, M., 1956. Remarks on some nonparametric estimates of a density function. Annals of Mathematical Statistics. 27(3), 832-837.

Zhang, S., 2010. A note on the performance of the gamma kernel estimators at the boundary. Statistics and Probability Letters, 80(7-8), 548-557. 